\title{
BLE-GSpeed: A New BLE-Based Dataset to Estimate User Gait Speed
}

\author{
Emilio Sansano-Sansano ${ }^{1, *(\mathbb{D},}$, Fernando J. Aranda ${ }^{2}{ }^{\mathbb{C}}$, Raúl Montoliu ${ }^{1}$ \\ and Fernando J. Álvarez ${ }^{2}$ (1) \\ 1 Institute of New Imaging Technologies, Universitat Jaume I, Avda. Vicente Sos Baynat S/N, \\ 12071 Castellón, Spain; montoliu@uji.es \\ 2 Sensory Systems Research Group, University of Extremadura, 06006 Badajoz, Spain; fer@unex.es (F.J.A.); \\ fafranco@unex.es (F.J.Á.) \\ * Correspondence: esansano@uji.es
}

Received: 9 November 2020; Accepted: 4 December 2020; Published: 7 December 2020

\begin{abstract}
To estimate the user gait speed can be crucial in many topics, such as health care systems, since the presence of difficulties in walking is a core indicator of health and function in aging and disease. Methods for non-invasive and continuous assessment of the gait speed may be key to enable early detection of cognitive diseases such as dementia or Alzheimer's disease. Wearable technologies can provide innovative solutions for healthcare problems. Bluetooth Low Energy (BLE) technology is excellent for wearables because it is very energy efficient, secure, and inexpensive. In this paper, the BLE-GSpeed database is presented. The dataset is composed of several BLE RSSI measurements obtained while users were walking at a constant speed along a corridor. Moreover, a set of experiments using a baseline algorithm to estimate the gait speed are also presented to provide baseline results to the research community.
\end{abstract}

Dataset: http:/ / doi.org/10.5281/zenodo.4261381.

Keywords: gait speed; public database; BLE-based technology

\section{Introduction}

Over the next 40 years, the percentage of people aged 60 and older is expected to rise from $10 \%$ to $22 \%$ of the total population [1]. This issue will pose a challenge for health care systems, especially considering that older people have more health-related issues and long-term care needs than the rest of society. In this context, cognitive decline and dementia are predicted to double their number of cases every 20 years globally. Health systems have not been oriented toward these needs and may have difficulties responding to the new demographic reality and the associated changes in population health. The presence of walking difficulties is a core indicator of health and function in aging and disease [2]. Gait control is problematic since it integrates multiple systems, including motor, perceptual, and cognitive processes. As any dysfunction in these systems leads to gait slowing, gait speed (GS) is a commonly used parameter in health care research. Because walking speed is a quick, reliable, sensitive, and easy measurement to perform [3-6], it is often included in clinical and epidemiological research studies [7-9], as a consistent risk factor for adverse outcomes in community-dwelling older people. In addition to sex and age, it is used to monitor older adults' functional capacity and forecast their age-related decline rate. The accuracy of predictions based on these three factors is generally similar to more elaborate models requiring many other health-related factors [10]. Several studies have confirmed an association linking gait speed with many significant health-related outcomes, including hospitalization, falls, nursing home placement, mobility disability, and cognitive diseases 
such as Alzheimer's disease [7,8,11]. Making better use of technology to stress disease prevention and early detection is key to meeting upcoming societies' needs. Future treatments could target cognitive diseases in their earliest stages, before irreversible brain damage or mental decline. Gait speed, age, and sex may offer the clinician tools to assess expected survival and tailor care goals in older adults.

Currently, the equipment used to perform GS measurements needs specialized personal to operate it; therefore, it is only used in clinical environments. For example, the kinetic system, developed by Microsoft, has been used to study the relationship between GS and other kinematic parameters with certain conditions [12,13]. Other vision-based techniques like deep cameras have also been used with similar results [14]. Many technologies have been used for the same task in in-home environments. For instance, ultrasound [3,15,16], passive infrared sensors (PIR) [17], and radio frequency $[4,18]$ have been widely used in this context, but only for research purposes. These systems can provide acceptable results, but they cannot identify different users in a multi-resident context, limiting their use. Inertial systems can offer a solution to the identification problem, but their precision is still very low [19].

Despite all the cited developments, there is no standard in-home GS measurement system for these kinds of environments. Therefore, more research is needed to face future healthcare systems to challenge and improve the monitoring of certain illnesses. More developments on each technology and new methods are needed to find the most suitable solution. Unfortunately, it is not always possible for researchers to build their experiments and work with them due to resource limitations. This problem could be solved with data results sharing through the means of the databases and data descriptors. Researchers worldwide can use Databases to test their methods in different environments and conditions without repeating others' experiments, which is time and cost consuming. Moreover, data sharing can create new research partnerships between teams from different institutions and disciplines with the same objectives and interests. For all these reasons, the use of databases has grown significantly in recent years.

Regarding GS measurement, there are several databases for the evaluation of different illnesses, like Parkinson [20], and Alzheimer [19], and their relation with GS. However, to the best of the authors' knowledge, there is only one database for technology evaluation in this field. More databases with a detailed description of the experimental set-up, the proposed system, and the results are needed to find a solution to the problem mentioned above.

This work's primary contribution is to introduce a new Bluetooth Low Energy (BLE) database to the research community to evaluate the GS in an in-home environment. The database is composed of BLE RSSI measurements from different wearable devices and different BLE beacons. This is, to the best of the authors' knowledge, the first work in this field using BLE as the main technology to evaluate the GS and among the first databases for technology evaluation. The use of this technology allows the system to identify the user while measuring the GS automatically. The experiments were carried out in a hallway at University Jaume I of Castellón in Spain. In these experiments, 13 users walked through the hallway at different speeds, varying from slow to fast, while wearing a wearable smartwatch device. The smartwatches recorded the received signal strength indicators (RSSI) of a set of BLE beacons placed in the ceiling. A set of ultrasonic sensors was used to compute each walk's average speed, used as ground truth, to obtain an accurate GS estimation to compare our system's estimations. Apart from the data and experimental environment description, a baseline for using this database is presented in this work. The database [21] contains the data from the experiments and the baseline source code.

The remainder of this work is organized as follows. In Section 2 related dataset and similar works with other databases are described. Section 3 explains the experimental set-up and the database metadata. Section 4 introduces the baseline for the gait speed determination as a how-to-use example. Finally, a discussion and the main conclusions are presented in Section 5. 


\section{Related Work}

The publication of databases has become very common in some research fields, especially when researchers cannot reproduce the experimental developments. Alongside the results, detailed information on the experiment characteristics and the test environment is vital in the databases' reproducibility and validation. In another context, where data is gathered from observation of real activities, databases could help understand and predict people's behavior. For example, in the post-COVID-19 era, databases can be used to develop tracking and location-aware systems to control the epidemic [22]. These databases are accompanied by the development of standardization formats and specialized tools like Zenodo and Kaggle [23], where the data are maintained for public access. Here, similar databases to the one presented in this work are described.

People's behavior in real complex environments has been studied using wearable BLE receivers carried by the users. BLE is a radio-frequency protocol communication, similar to Wi-Fi, and developed for short communication in the context of the Internet of Things (IoT). For example, Sikeridis et al. published the measurements from a set of Raspberry Pi boards measuring the RSSI from 46 students' wearables, smartwatches and smartphones, in a multi-floor university building during their daily activities during one-month [24]. Similarly, Tóth and Jamas [25] provide similar databases with measurements in similar environments from different radio-frequency wireless networks, such as $\mathrm{BLE}, \mathrm{Wi}-\mathrm{Fi}$, and traditional Bluetooth. The same kind of experiments has been repeated in controlled environments where the data, or at least part of it, can be labeled for further analysis. Byrne et al. [26] present a database with data from multiple houses; in each one, a participant wore a custom wearable device with an accelerometer, BLE, and camera. A set of image codes distributed over the houses and recorded by the camera are used to label the measurements to the label de information. In [27] Iqba et al. propose a BLE passive fingerprinting system that can locate persons inside a medical building. The training and test data were published in a database [28]. This is becoming a common practice in fingerprinting positioning where the environment, methodology, and methods can change the same positioning algorithm's results. There are several fingerprinting databases using Wi-Fi RSSI measurements. The first database of this kind is the UJIIndoorLoc [29], which presents RSSI measures in a university environment taken by more than 20 users and 25 devices. Similar databases have been published for congress competitions in the development of indoor positioning systems [30,31].

Since the biggest smartphone software developers have limited the scanning times, BLE fingerprinting positioning has become a suitable alternative. Mendoza et al. [32] present an RSS BLE database with measurements from two different scenarios, a laboratory where multiple beacon configurations were tested, and an university library where data from various users and devices were collected. The database is accompanied by the necessary tools to load and work with it. Similarly, Aranda et al. [33] provide a BLE RSS database with beacons with multiple slots deployed in three different indoor/outdoor environments. The database can be found in the Zenodo repository [34], with a complete description of the experimental environment. Baronti et al. [35] repeat the same fingerprinting experiment with different configurations for active and passive fingerprinting, using the same real point in both cases. Moreover, they add data from experiments to explore the tracking and social interaction between users in the facility.

Some researchers have gathered gait and kinematic information from healthy patients. This information can be used for health-researchers as a baseline for compare their results when investigating specific illnesses. For example, Fukuchi et al. [36] developed a database with detailed kinematic information of 42 healthy volunteers; similarly, Schreiber et al. [37] performed a similar experiment measuring the GS in 50 free-injury participants. Custom high-precision inertial sensors have also been used in [38], focusing on the age difference, and in [39], with the focus on detecting changes in the gait mechanics. All these databases are focused on the health relation between GS and other parameters. To the best of the authors' knowledge, only one publicly available database focuses on evaluating technology for the GS measurement. Chapron et al. [40] combined a set of PIR sensors with a BLE indoor positioning system to detect the closest user to the PIR sensors. 
With this solution, the system can automatically label the GS measurements to the correct user in a multi-resident environment. Similarly, the database presented in this work is also designed for GS evaluation, but using a completely new approach in this field.

Finally, more databases for evaluating different technologies in different environments are needed to find a standard solution for the GS measurement in the in-home environment. A summary with some of the most relevant available databases is presented in Table 1, where the references to the databases and their associated works can be found.

Table 1. References to some of the most relevant available databases for people monitoring, fingerprinting and GS.

\begin{tabular}{cccccc}
\hline Database & People Monitoring & Wi-Fi Fingerprinting & Bluetooth Fingerprinting & GS Monitoring & GS Evaluation \\
\hline Works & {$[24-27]$} & {$[29-31,41,42]$} & {$[32,33,35]$} & {$[36,38,39]$} & {$[40]$} \\
Databases & {$[28,43-45]$} & {$[46,47]$} & {$[34,48,49]$} & {$[50-52]$} & {$[53]$} \\
\hline
\end{tabular}

\section{Data}

The data collection process was performed in a hallway at the Universitat Jaume I in Castellón, Spain. Figure 1 shows a view of the deployment. A total of 20 beacons were mounted on the hallway's ceiling, with a separation of $30 \mathrm{~cm}$ between them. We used two different models alternatively, the iBKS 105 model [54] and the iBKS plus model [55], so the separation between two beacons of the same model was $60 \mathrm{~cm}$.

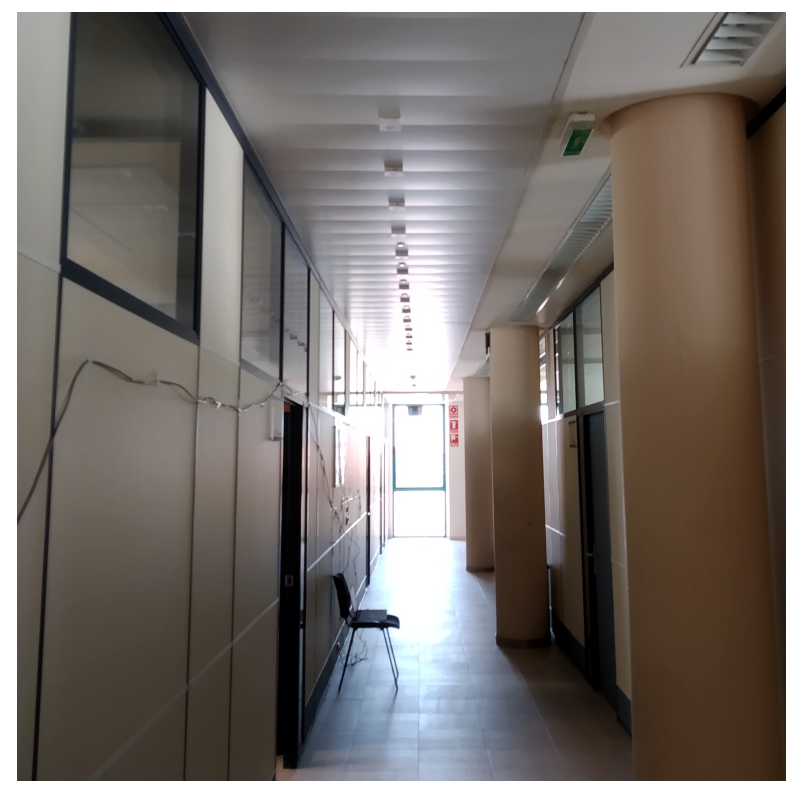

Figure 1. A view of the beacons deployed in the ceiling of the corridor.

Figure 2 shows a scheme of: (a) the models and distribution of the ceiling-mounted beacons, and (b) the infrastructure used to acquire the ground truth and the data. To determine the user's actual speed we used five ultrasonic sensors HC-SR04 attached to the wall at the height of $0.7 \mathrm{~m}$ and with a separation of $3.5 \mathrm{~m}$ between them, covering a total distance of $14 \mathrm{~m}$. The sensors were connected to an Arduino UNO board that recorded the readings and associated timestamps for each sensor during the experiments. This information was stored in real time in a laptop connected through the serial port to the Arduino board and synchronized through an NTP server to an absolute time frame of reference. On the other hand, the smartwatches used to capture the RSSI signals also use the server as a reference to synchronize their scan results with the user's position captured by the ultrasound sensors. 
For a particular walk, the wall-mounted sensors provide the timestamps at which the user passed in front of them. For each pair of sensors $(i$ and $j)$, the speed of the user can be obtained as follows:

$$
v_{i, j}=\frac{d_{i j}}{\left|t_{i}-t_{j}\right|}
$$

where $v_{i, j}$ is the speed at which the user moved from sensor $i$ to sensor $j, d_{i j}$ is the distance between sensors, and $t_{i}$ and $t_{j}$ are the timestamps at with the user passed in front of sensors $i$ and $j$, respectively. Considering only pairs of consecutive sensors, we obtain four speed values for each walk. The resultant speed is calculated as the average of all four measurements, but only when their discrepancies are less than $5 \mathrm{~cm} / \mathrm{s}$, since we only want to keep those walks executed at a constant speed.

(a)

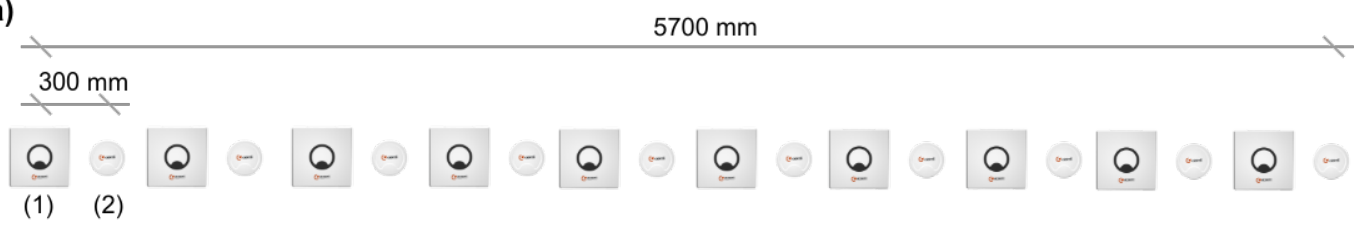

(b) $3500 \mathrm{~mm}$ $3500 \mathrm{~mm}$ $3500 \mathrm{~mm}$ $3500 \mathrm{~mm}$

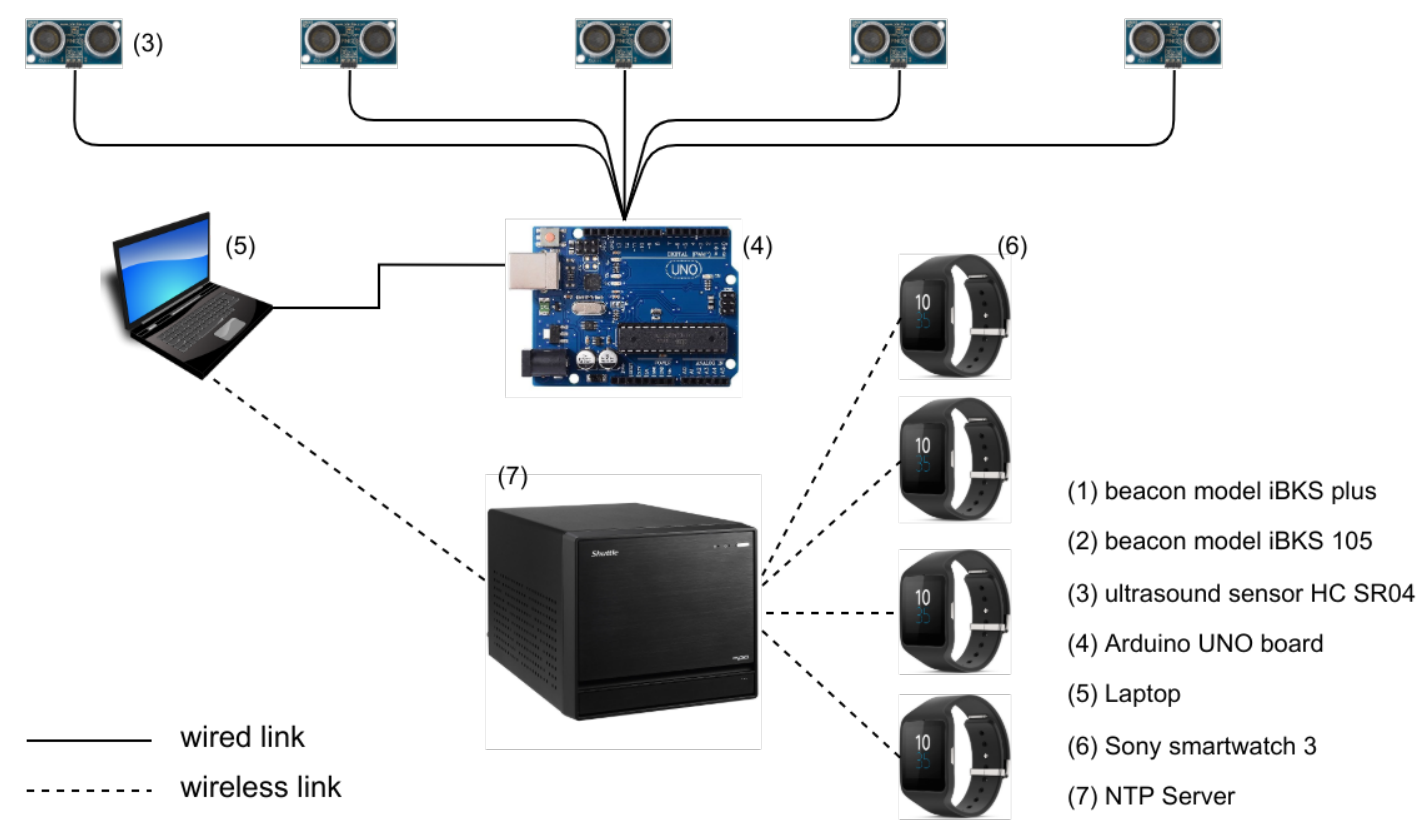

Figure 2. Scheme of the set-up used to capture the data. (a) disposition of the beacons on the ceiling, (b) general scheme of the setup used during the data acquisition process.

A total of 13 subjects, 11 males and 2 females, aged between 18 and 55, performed several walks in both directions along the hallway. The subjects were instructed to keep their walking speed constant during the process. Each user completed several walks at different speeds, from very slow to fast. Figure 3 shows: (a) the distributions of speeds recorded by each user, (b) the number of walks recorded by each user, and (c) the overall distribution of speeds in the data set. The minimum speed recorded was $0.43 \mathrm{~m} / \mathrm{s}$ and the maximum $1.87 \mathrm{~m} / \mathrm{s}$. 

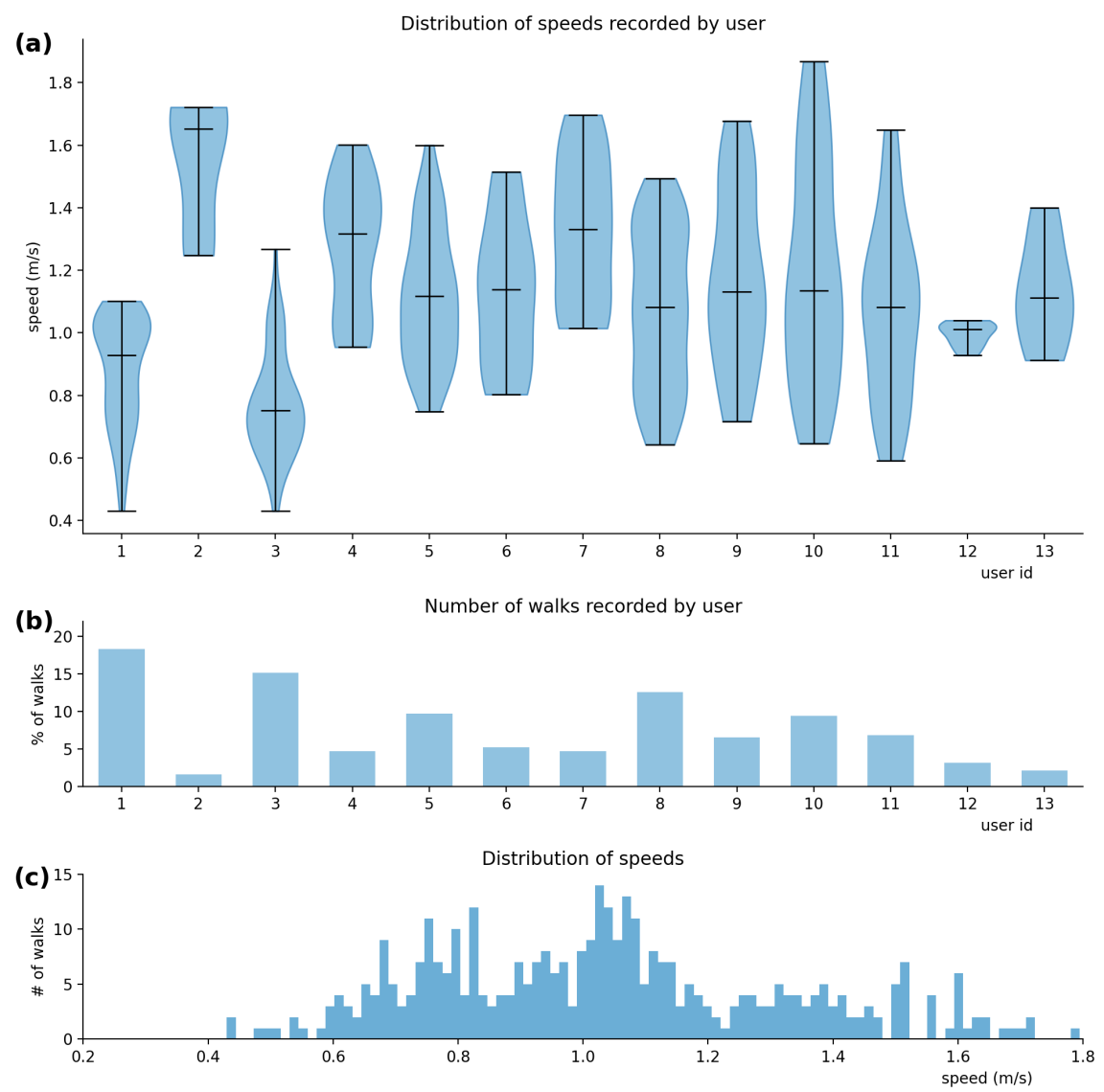

Figure 3. (a) Violin plots of the speeds by user. (b) number of walks by user. (c) general distribution of speeds in the dataset.

To increase the volume of data recorded, users wore four smartwatches during the acquisition process, two on each wrist. The model used in the experiments was the Sony smartwatch 3, which runs Android as its operating system. An application installed in the smartwatches performed continuous Bluetooth scans, at the maximum frequency allowed by the operating system, and stored the results for later post-processing. Once all the data has been processed, each register of the resultant data set represents a scan result, and is composed of the following fields:

- mac. The MAC address of the detected beacon.

- $\quad r s s i$. The RSSI value obtained for the beacon.

- device. A four-character descriptor for the smartwatch that performed the scan.

- timestamp. The timestamp at which the scan was received.

- $\quad$ user. The id of the user that was performing the experiment.

- direction. A number (0 or 1$)$ indicating the direction of the walk.

- $\quad$ walk_id. A number that identifies each walk.

- $\quad$ speed. The actual speed of the user, in $\mathrm{m} / \mathrm{s}$.

Overall, the final data set contains a total of 382 walks. Figure 4a compares the distributions of the scanned RSSI values for each device, as well as the average number of RSSI values acquired per second. For all devices, the scanning rate oscillates between 40 and 50 results per second. During the acquisition process, one of the beacons started to malfunction and finally stopped working, so its data has not been considered. Taking into account that there were 19 functioning beacons, this represents that the advertising signal of a particular beacon is detected roughly 2.4 times per second by each device. Since the beacons were programmed to advertise at an interval of $100+0-10 \mathrm{~m} / \mathrm{s}$, the expected 
rate should be around 9 values per second. This discrepancy is due to the fact that the smartwatches' scanning rate cannot be directly set but is controlled by their operating system and designed to preserve battery life. With regards to the distribution of signals detected by each device, there are no significant differences. Median values for all devices are in the interval $-70 \mathrm{dBm}$ to $-75 \mathrm{dBm}$. Some devices, especially a 650 but also $14 \mathrm{df}$, seem to have a broader range of detection, capturing RSSI values in the range $-30 \mathrm{dBm}$ to $-105 \mathrm{dBm}$, while the remaining two devices only report RSSI values in the range $-45 \mathrm{dBm}$ to $-105 \mathrm{dBm}$.
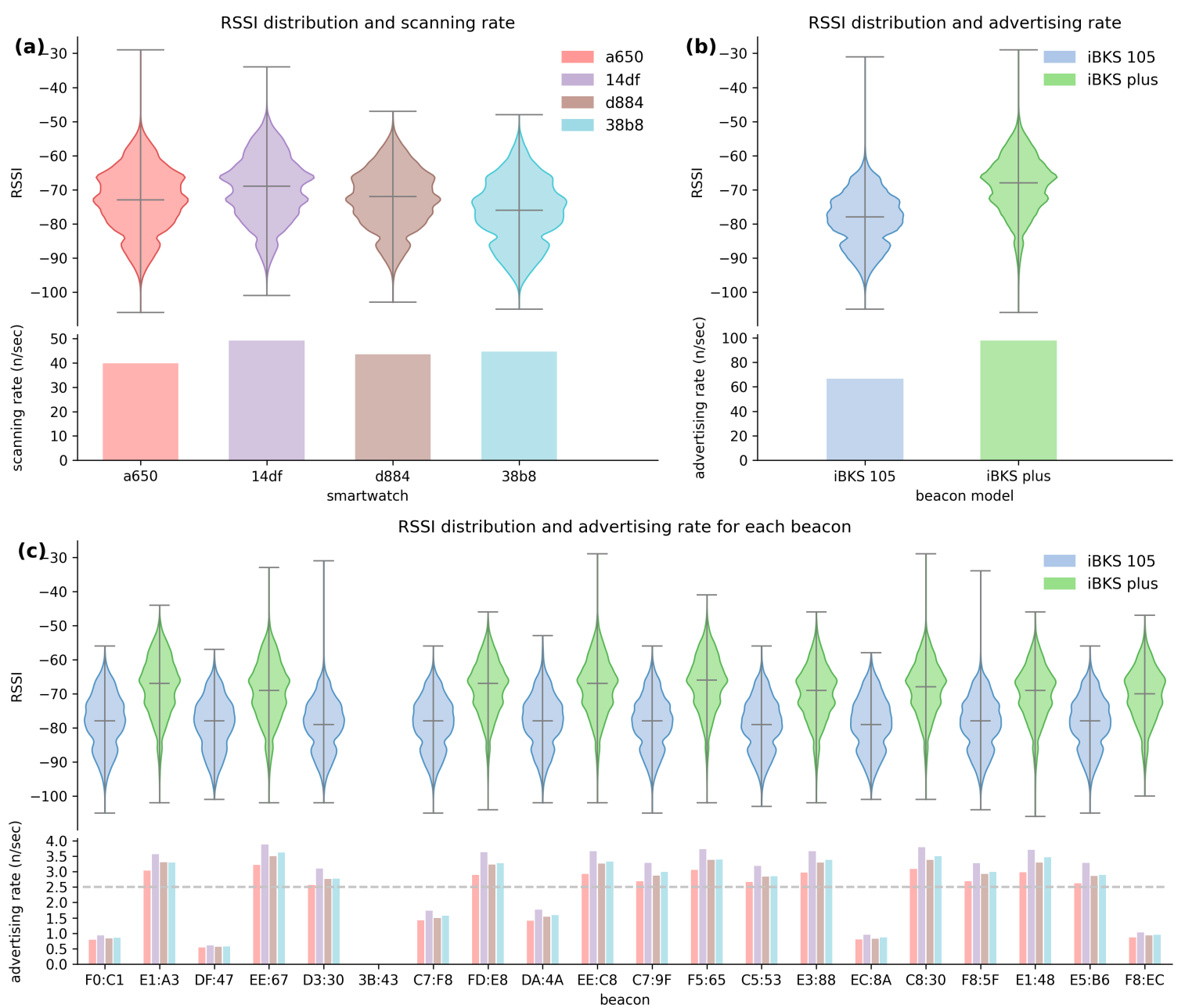

Figure 4. (a) distribution and scanning rate of the smartwatches. (b) RSSI distribution and advertising rate of the beacons (by model). (c) RSSI distribution and advertising rate for each beacon.

With respect to the beacons' characteristics, Figure $4 \mathrm{~b}$ shows the distribution of RSSI values segmented by beacon model, and the number of beacons of each model detected per second. Beacons of model $i B K s$ plus are distinctly detected with a stronger RSSI value that the beacons of model $i B K S$ 105. Besides their disparate emitting power, the shape of the distribution of the RSSI values is also significantly different. Both models are based on the same chipset (Nordic Semiconductors nrf51822), so the differences in performance might be due to different batteries (a $1000 \mathrm{mAh}$ CR2477 coin cell battery for the 105 model, four AA alkaline batteries with a total capacity of $5000 \mathrm{mAh}$ for the plus model) and/or their different casing and shape. BLE chipsets can demand a peak of $20 \mathrm{~mA}$ when transmitting. Coin cell batteries are greatly affected by large current draws [56], while alkaline batteries can handle larger currents. Figure $4 \mathrm{c}$ shows a comparison of the distribution of RSSI values for each beacon, as well as of their effective rate or advertisement, this is, for a given smartwatch, the number of scans per second in which the beacon is present. As shown in Figure $4 b$, the differences between 
advertising RSSI values coming from distinct models are clear and consistent. With respect to their advertising rates, even though all models have been set up with an advertising interval of $100 \mathrm{~ms}$, only five out of ten model 105 beacons can reach a detection rate superior to 2.5 scans per second. On the other hand, all model plus beacons except one are detected at a rate greater than 2.5 scans per second. The detection rate segmented by smartwatch and beacon is consistent with Figure $4 \mathrm{a}$, being $14 \mathrm{df}$ the smartwatch with the highest scanning rate for all beacons.

\section{Experiments}

This section aims to describe a method to determine the user's speed gait using the presented dataset. It illustrates a simple case of use that can be easily replicated so that the results may serve as a future baseline for researchers in this topic. The methods used to achieve these results are straightforward, and the implementation has been made publicly available.

\subsection{Gait Speed Determination}

The RSSI value provided by radio-frequency modules represents an indication of the power strength of the transmitter signal perceived by the receiver node. The RSSI value received at a particular location can be modeled as a function of the logarithmic distance between the receiver and the emitter, plus some parameters related to the environment properties and the devices' characteristics [57]. This analytical model allows estimating the position of a device, the scanning node, knowing the received RSSI value data and the emitting node's position. The path loss model reflects the relationship between the signal strength and the distance to the emitter:

$$
R S S I=R S S I_{0}-10 \gamma \log _{10} \frac{d}{d_{0}}+X_{g}
$$

where:

- $\quad$ RSSI is the received signal strength at a distance $d$ from the beacon.

- $R S S I_{0}$ is the received signal strength at the reference distance $(1 \mathrm{~m})$ from the beacon.

- $d$ is the distance between the receiver and the beacon.

- $d_{0}$ is the reference distance $(1 \mathrm{~m})$

- $X_{g}$ is a random variable with zero mean, reflecting the attenuation (in decibel) caused by fading, multipath effect, etc.

- $\quad \gamma$ is the path loss exponent, whose value is normally in the range of 2 to 6 . The actual value depends on environmental characteristics.

Figure 5a shows the theoretical evolution of the RSSI signal when a user wearing a receiver device walks at a constant speed along a straight line which passes below a BLE beacon. We consider the receiver moving at a constant speed $v$ in the presence of a ceiling-mounted emitting device (see Figure $5 b$ ), located at a distance $d$. Figure $5 c$ shows a real example of RSSI data received from the emitter when the user follows the path at a constant speed. It also shows a path loss curve fitted to the data. Even though perceived RSSI signals are subject to noise due to interferences, multipath effects, signal fluctuations, overlapping channels and other environmental characteristics, the maximum value received during the walk is likely to happen when the receiver is in the nearest point with respect to the emitter, this is, when the user is passing beneath the beacon. 
(a)
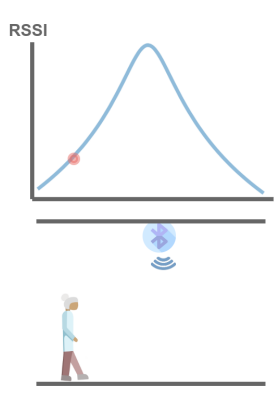

(b)

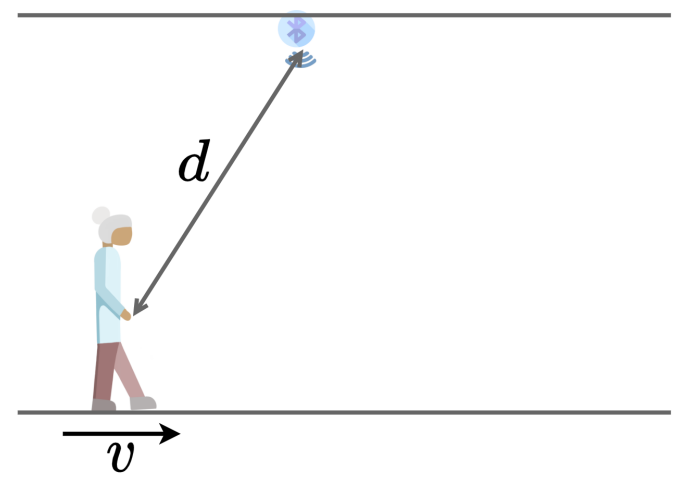

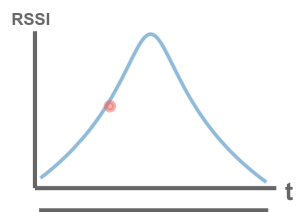
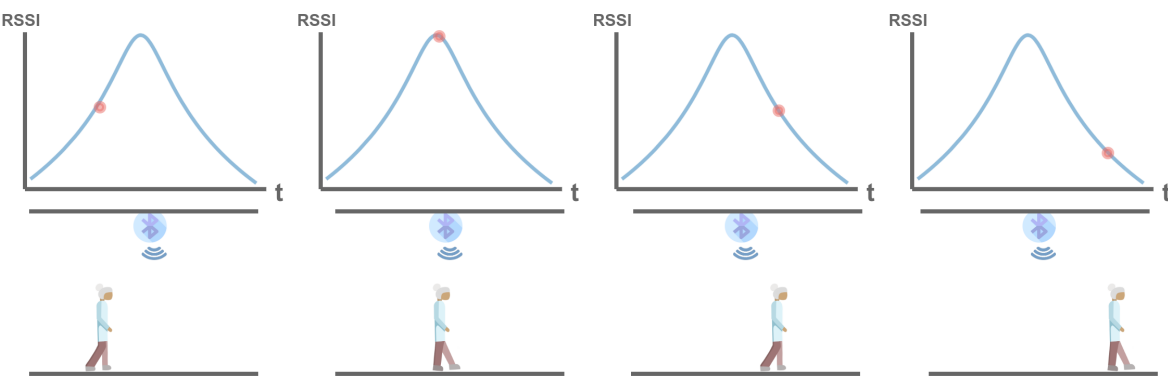

(c)

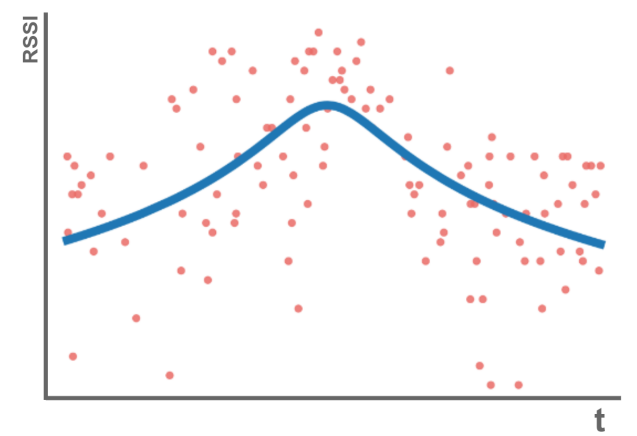

Figure 5. (a) Theoretical evolution of the perceived RSSI value during a walk. (b) Distance between the smartwatch and the beacon. (c) Theoretical (blue line) and real (red dots) RSSI values perceived during a walk.

To estimate the gait speed of the user, we performed the following process:

1. For each walk, smartwatch and mac (beacon), we find the timestamp $\widehat{t}$ at which the maximum RSSI value has been detected. We do so in two different ways; by looking at the raw data and applying a 13 point moving average and finding the maximum point in the smoothed version of the RSSI data. We tried different values for the window length, in the range between 3 and 25, obtaining the best results for a window length of 13 measurements.

2. For two given beacons $i, j$, separated by a distance $d_{i j}$, and with $\widehat{t}_{i}, \widehat{t}_{j}$ being the estimated timestamps at which the user walked below them, the speed of the receiver $\widehat{v_{i j}}$ can be estimated as follows:

$$
\widehat{v}_{i j}=\frac{d_{i j}}{\left|\widehat{t}_{i}-\widehat{t}_{j}\right|}
$$

3. In the general case, when there are more than two beacons installed, the speed can be estimated as the average of the values obtained for each pair. Given a set of $k$ beacons, the speed of the device is calculated as follows:

$$
\hat{v}=\frac{\sum \hat{v}_{i j}}{n}
$$

where $j \in[1,2, \ldots, k-1], i \in[j+1, j+2, \ldots, k]$, and $n=\left(\begin{array}{l}k \\ 2\end{array}\right)$.

4. The speed estimation obtained for each pair is only taken into account when it is comprised in the interval $0.2<\hat{v}<1.8$. This is not just because we want to consider only results that correspond to a feasible user speed, but also because the low scanning rate of the smartwatches may produce insufficient data to achieve a good estimation, and can generate artifact results that may not represent a proper approximation of the actual speed of the user. 


\subsection{Results}

The results obtained for each smartwatch and beacon model are summarized in Figure 6. The plot shows the average error in $\mathrm{m} / \mathrm{s}$ for the gait speed estimation for each smartwatch, for each beacon model, and for several beacons ranging from 2 to 10 .

The first conclusion we draw from the results is that using raw data to estimate the gait speed provides a very uniform average error (see the blue continuous line in Figure 6), which is independent of the number of beacons considered. There is a slight improvement in accuracy when using three beacons instead of two, but adding more beacons does not significantly reduce the error, which remains around $0.2 \mathrm{~m} / \mathrm{s}$ regardless of the beacon model or the smartwatch considered. On average, the results obtained when using beacons of model $i B K S$ plus are slightly better than those obtained when using beacons of model $i B K S$ 105, but in general, results are very similar.
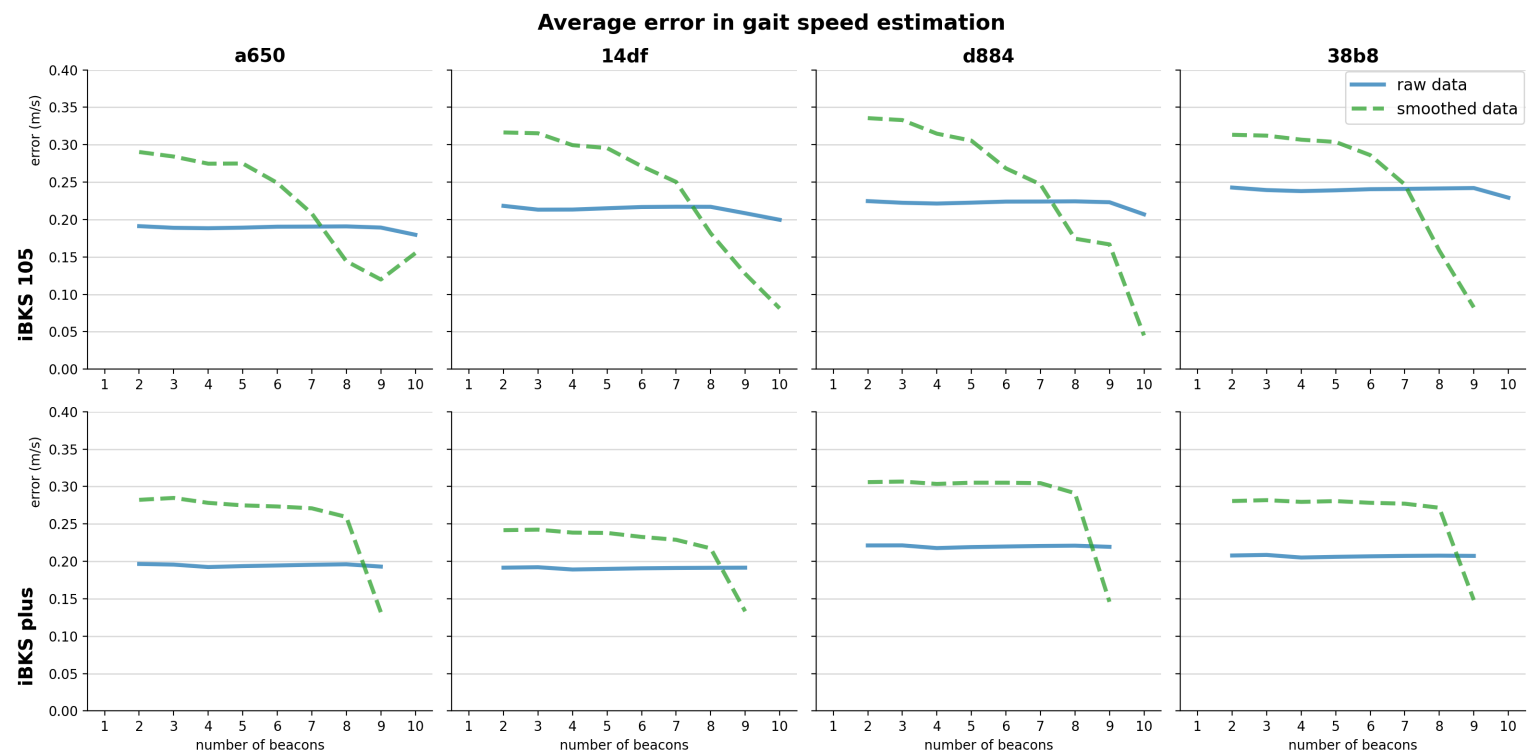

Figure 6. Error in m/s of gait speed estimation for each smartwatch and beacon model.

On the other hand, results look very different when using smoothed data to estimate the timestamp of the maximum RSSI value received during the walks. The green and discontinuous lines in Figure 6 show a distinctive behavior from the previous case. Now the error in the estimation is strongly dependent on the number of beacons considered. When using only two beacons, the error is always greater than when using raw data, but it decreases as the number of beacons increases. This is especially evident when considering only beacons of model $i B K S$ 105. In this case, the error starts at around $0.3 \mathrm{~m} / \mathrm{s}$ for 2 beacons and goes below $0.1 \mathrm{~m} / \mathrm{s}$ for 10 beacons. The same behavior occurs for model $i B K S$ plus, but with a less pronounced decrease of the error, which arrives at a value of around $0.15 \mathrm{~m} / \mathrm{s}$ when we consider 9 beacons.

Mixing different models of beacons (Figure 7) seems not to influence accuracy when using only raw data. When using smoothed data, results are consistently worse, and they do not improve substantially when the number of beacons increases. The fact that we obtain worse results when mixing beacon models may be due to their different characteristics regarding their RSSI distribution (Figure $4 b$ ). Tackling this would probably require a specific approach for each model with respect to the technique used to perform the data smoothing. 


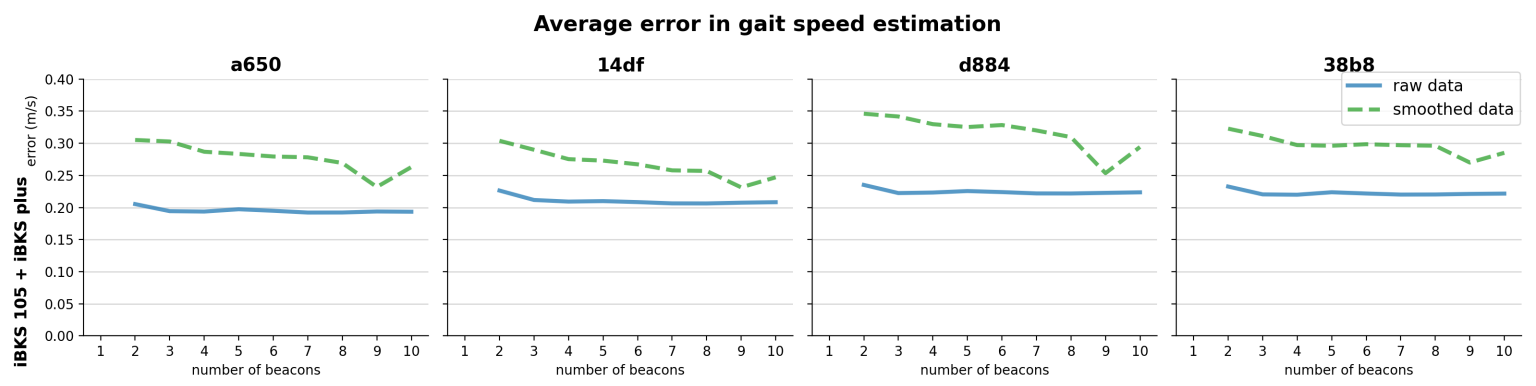

Figure 7. Error in $\mathrm{m} / \mathrm{s}$ of gait speed estimation for each smartwatch and a mix of both beacon models.

Better results are obtained when we aggregate the data from all the smartwatches and consider beacons of model $i B K S$ plus (Figure 8 ). In this case, the average error is around $0.17 \mathrm{~m} / \mathrm{s}$. Again, results obtained with smoothed data are generally worse than those obtained using raw data, except in cases where the number of beacons of the same model is large.

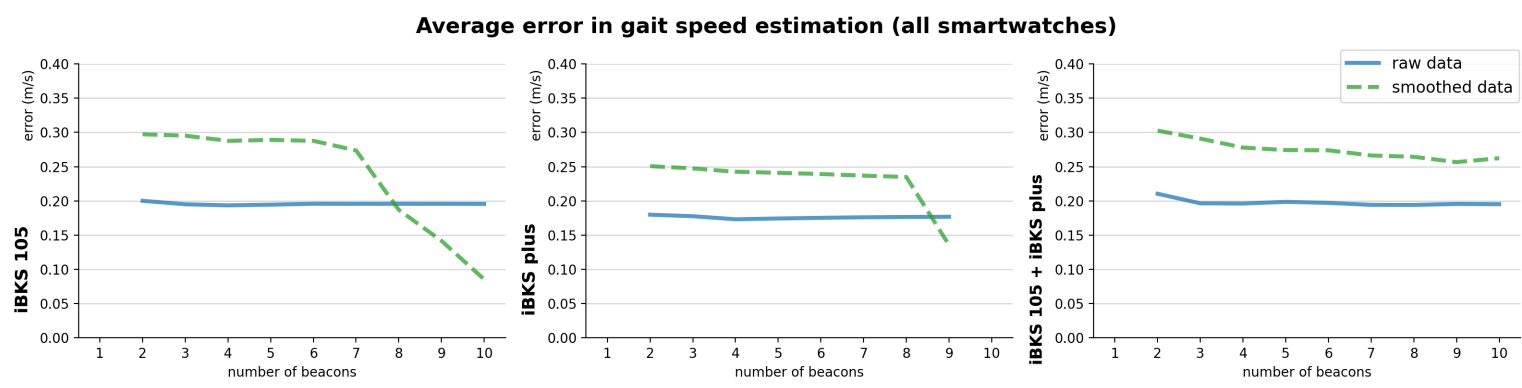

Figure 8. Error in $\mathrm{m} / \mathrm{s}$ of gait speed estimation considering the aggregated data from all smartwatches.

Table 2 shows a summary of the results obtained considering all the smartwatches. The best results are obtained when using 10 beacons of model iBKS 105 and performing a 13-point moving average smoothing on the raw data. In general, smoothing provides better results when using a large number of same-model beacons. On the contrary, when the number of beacons is limited, estimating the gait speed using the raw data is the best approach.

Table 2. Summary of the best results obtained

\begin{tabular}{lccc}
\hline Beacon Model & Method & Error $(\mathbf{m})$ & \# Beacons \\
\hline iBKS 105 & raw data & 0.1935 & 4 \\
iBKS 105 & smoothed data & 0.0855 & 10 \\
iBKS plus & raw data & 0.1732 & 4 \\
iBKS plus & smoothed data & 0.1357 & 9 \\
mixed & raw data & 0.1928 & 8 \\
mixed & smoothed data & 0.2566 & 9 \\
\hline
\end{tabular}

\section{Conclusions}

Intending to support the rising success of data-driven approaches in several fields, such as computer science, engineering, or healthcare, the availability of specialized datasets has become decisive. Although the volume of available data grows exponentially from an expanding diversity of data sources, getting curated data ready for application is a costly and time-consuming process.

In this work, we present a new Bluetooth Low Energy-based dataset for gait speed estimation. This is the first BLE-based dataset for gait speed estimation that is publicly available to the best of our knowledge,. The dataset was created by using a large number of devices and actors, trying to represent a wide variety of gait speeds and styles of walking. We explore the use of the dataset by 
analyzing its properties and showcasing some relevant results, with the purpose that this dataset may be useful to researchers and practitioners that may use it to experiment and test their algorithms.

Our current and future work aims to develop more automated and general ways of acquiring user's speed data, with an eye on more familiar environments such as homes.

\section{Reproducibility}

The code to reproduce all the plots and experiments described in this work is publicly available at https://github.com/esansano/ble-gspeed.

Author Contributions: E.S.-S. has been in charge of the conceptualization, methodology and manuscript writing. F.J.A. assisted in the design of the experimental set-up and the writing of the manuscript. R.M. helped the creation of the experimental set-up, the writing of the manuscript, and guided the research work. Finally, F.J.Á. guided the research work, reviewed the contents and approved the paper. All authors have read and agreed to the published version of the manuscript.

Funding: This work was supported in part by the Spanish Ministry of Economy and Competitiveness through projects MICROCEBUS (RTI2018-095168-B-C53/C54), REPNIN+ (TEC2017-90808-REDT) and in part by the Regional Government of Extremadura and the European Regional Development Fund through project GR18038 and the Regional Government of Valencian Community by project AICO/2020/046.

Acknowledgments: Authors want to agree to all volunteers who perform the walks included in the dataset.

Conflicts of Interest: The authors declare no conflict of interest.

\section{Abbreviations}

The following abbreviations are used in this manuscript:

BLE Bluetooth Low Energy

GS Gait Speed

RSSI Received Signal Strength Indicator

PIR Passive Infrared Sensors

NTP Network Time Protocol

\section{References}

1. Bloom, D.E.; Chatterji, S.; Kowal, P.; Lloyd-Sherlock, P.; McKee, M.; Rechel, B.; Rosenberg, L.; Smith, J.P. Macroeconomic implications of population ageing and selected policy responses. Lancet 2015, 385, 649-657. [CrossRef]

2. Peel, N.M.; Kuys, S.S.; Klein, K. Gait speed as a measure in geriatric assessment in clinical settings: A systematic review. J. Gerontol. Ser. A 2013, 68, 39-46. [CrossRef] [PubMed]

3. Ferre, X.; Villalba-Mora, E.; Caballero-Mora, M.A.; Sanchez, A.; Aguilera, W.; Garcia-Grossocordon, N.; Nuñez-Jimenez, L.; Rodríguez-Mañas, L.; Liu, Q.; del Pozo-Guerrero, F. Gait speed measurement for elderly patients with risk of frailty. Mob. Inf. Syst. 2017, 2017, 1310345. [CrossRef]

4. Hsu, C.Y.; Liu, Y.; Kabelac, Z.; Hristov, R.; Katabi, D.; Liu, C. Extracting gait velocity and stride length from surrounding radio signals. In Proceedings of the $2017 \mathrm{CHI}$ Conference on Human Factors in Computing Systems, Denver, CO, USA, 6-11 May 2017; pp. 2116-2126.

5. Qian, K.; Wu, C.; Yang, Z.; Liu, Y.; Jamieson, K. Widar: Decimeter-level passive tracking via velocity monitoring with commodity Wi-Fi. In Proceedings of the 18th ACM International Symposium on Mobile Ad Hoc Networking and Computing, Chennai, India, 10-14 July 2017; pp. 1-10.

6. Zhang, F.; Chen, C.; Wang, B.; Liu, K.R. WiSpeed: A statistical electromagnetic approach for device-free indoor speed estimation. IEEE Internet Things J. 2018, 5, 2163-2177. [CrossRef]

7. Cesari, M.; Kritchevsky, S.B.; Penninx, B.W.; Nicklas, B.J.; Simonsick, E.M.; Newman, A.B.; Tylavsky, F.A.; Brach, J.S.; Satterfield, S.; Bauer, D.C.; et al. Prognostic value of usual gait speed in well-functioning older people-Results from the Health, Aging and Body Composition Study. J. Am. Geriatr. Soc. 2005, 53, 1675-1680. [CrossRef] 
8. Hackett, R.A.; Davies-Kershaw, H.; Cadar, D.; Orrell, M.; Steptoe, A. Walking speed, cognitive function, and dementia risk in the English longitudinal study of ageing. J. Am. Geriatr. Soc. 2018, 66, 1670-1675. [CrossRef] [PubMed]

9. Guralnik, J.M.; Ferrucci, L.; Pieper, C.F.; Leveille, S.G.; Markides, K.S.; Ostir, G.V.; Studenski, S.; Berkman, L.F.; Wallace, R.B. Lower extremity function and subsequent disability: Consistency across studies, predictive models, and value of gait speed alone compared with the short physical performance battery. J. Gerontol. Ser. A Biol. Sci. Med Sci. 2000, 55, M221-M231. [CrossRef] [PubMed]

10. Studenski, S.; Perera, S.; Patel, K.; Rosano, C.; Faulkner, K.; Inzitari, M.; Brach, J.; Chandler, J.; Cawthon, P.; Connor, E.B.; et al. Gait speed and survival in older adults. JAMA 2011, 305, 50-58. [CrossRef] [PubMed]

11. Sayers, S.P.; Jette, A.M.; Haley, S.M.; Heeren, T.C.; Guralnik, J.M.; Fielding, R.A. Validation of the late-life function and disability instrument. J. Am. Geriatr. Soc. 2004, 52, 1554-1559. [CrossRef]

12. Liu, L.; Mehrotra, S. Patient walk detection in hospital room using Microsoft Kinect V2. In Proceedings of the 2016 38th Annual International Conference of the IEEE Engineering in Medicine and Biology Society (EMBC), Orlando, FL, USA, 16-20 August 2016; Volume 2016, pp. 4395-4398. [CrossRef]

13. Songra, R.; Lockhart, T. Agreement in Gait Speed from Smartphone and Stopwatch for Five Meter Walk in Laboratory and Clinical Environments. Biomed. Sci. Instrum. 2014, 50, 254-264.

14. Stuck, A.K.; Bachmann, M.; Füllemann, P.; Josephson, K.R.; Stuck, A.E. Effect of testing procedures on gait speed measurement: A systematic review. PLoS ONE 2020, 15, e0234200. [CrossRef] [PubMed]

15. Weir, R.; Childress, D. A new method of characterising gait using a portable, real-time, ultrasound ranging device. In Proceedings of the 19th Annual International Conference of the IEEE Engineering in Medicine and Biology Society. 'Magnificent Milestones and Emerging Opportunities in Medical Engineering' (Cat. No.97CH36136), Chicago, IL, USA, 30 October-2 November 1997; Volume 4, pp. 1810-1812.

16. Pinson, S.; Holland, C.W. Relative velocity measurement from the spectral phase of a match-filtered linear frequency modulated pulse. J. Acoust. Soc. Am. 2016, 140, EL191-EL196. [CrossRef] [PubMed]

17. Hagler, S.; Austin, D.; Hayes, T.L.; Kaye, J.; Pavel, M. Unobtrusive and ubiquitous in-home monitoring: A methodology for continuous assessment of gait velocity in elders. IEEE Trans. Biomed. Eng. 2009, 57, 813-820. [CrossRef] [PubMed]

18. Wang, W.; Liu, A.; Shahzad, M. Gait recognition using wifi signals. In Proceedings of the UbiComp '16: Proceedings of the 2016 ACM International Joint Conference on Pervasive and Ubiquitous, Heidelberg, Germany, 12-14 September 2016; pp. 363-373. [CrossRef]

19. Keppler, A.M.; Nuritidinow, T.; Mueller, A.; Hoefling, H.; Schieker, M.; Clay, I.; Böcker, W.; Fürmetz, J. Validity of accelerometry in step detection and gait speed measurement in orthogeriatric patients. PLoS ONE 2019, 14, e0221732. [CrossRef] [PubMed]

20. Beck, Y.; Herman, T.; Brozgol, M.; Giladi, N.; Mirelman, A.; Hausdorff, J. SPARC: A new approach to quantifying gait smoothness in patients with Parkinson's disease. J. Neuroeng. Rehabil. 2018, 15. [CrossRef] [PubMed]

21. Sansano-Sansano, E.; Aranda, F.J.; Montoliu, R.; Álvarez, F.J. GSPEED—BLE-Based Gait Speed Dataset. 2020. Available online: https:/ / doi.org/10.5281/zenodo.4261381 (accessed on 6 December 2020). [CrossRef]

22. Shubina, V.; Holcer, S.; Gould, M.; Lohan, E.S. Survey of Decentralized Solutions with Mobile Devices for User Location Tracking, Proximity Detection, and Contact Tracing in the COVID-19 Era. Data 2020, 5, 87. [CrossRef]

23. Kaggle: Your Machine Learning and Data Science Community. Available online: https://www.kaggle.com/ (accessed on 3 November 2020).

24. Sikeridis, D.; Papapanagiotou, I.; Devetsikiotis, M. BLEBeacon: A Real-Subject Trial Dataset from Mobile Bluetooth Low Energy Beacons. arXiv 2018, arXiv:1802.08782.

25. Tóth, Z.; Tamás, J. Miskolc IIS hybrid IPS: Dataset for hybrid indoor positioning. In Proceedings of the 2016 26th International Conference Radioelektronika (RADIOELEKTRONIKA), Kosice, Slovakia, 19-20 April 2016; pp. 408-412.

26. Byrne, D.; Kozlowski, M. Residential Wearable RSSI and Accelerometer Measurements with Detailed Annotations. Sci. Data 2019. [CrossRef]

27. Iqbal, Z.; Luo, D.; Henry, P.; Kazemifar, S.; Rozario, T.; Yan, Y.; Westover, K.; Lu, W.; Nguyen, D.; Long, T.; et al. Accurate real time localization tracking in a clinical environment using Bluetooth Low Energy and deep learning. PLoS ONE 2018, 13, e0205392. [CrossRef] 
28. Byrne, D.; Kozlowski, M. Residential Wearable RSSI and Accelerometer Measurements with Detailed Annotations. 2018. Available online: https://figshare.com/articles/Residential_Wearable_RSSI_and_ Accelerometer_Measurements_with_Detailed_Annotations / 6051794 (accessed on 9 July 2020). [CrossRef]

29. Torres-Sospedra, J.; Montoliu, R.; Martínez-Usó, A.; Avariento, J.P.; Arnau, T.J.; Benedito-Bordonau, M.; Huerta, J. UJIIndoorLoc: A new multi-building and multi-floor database for WLAN fingerprint-based indoor localization problems. In Proceedings of the 2014 International Conference on Indoor Positioning and Indoor Navigation (IPIN), Busan, Korea, 27-30 October 2014; pp. 261-270.

30. Torres-Sospedra, J.; Jiménez, A.; Knauth, S.; Moreira, A.; Beer, Y.; Fetzer, T.; Ta, V.C.; Montoliu, R.; Seco, F.; Mendoza-Silva, G.; et al. The Smartphone-Based Offline Indoor Location Competition at IPIN 2016: Analysis and Future Work. Sensors 2017, 17, 557. [CrossRef]

31. Montoliu, R.; Sansano, E.; Torres-Sospedra, J.; Belmonte, O. IndoorLoc platform: A public repository for comparing and evaluating indoor positioning systems. In Proceedings of the 2017 International Conference on Indoor Positioning and Indoor Navigation (IPIN), Sapporo, Japan, 18-21 September 2017; pp. 1-8.

32. Mendoza-Silva, G.; Matey-Sanz, M.; Torres-Sospedra, J.; Huerta, J. BLE RSS Measurements Dataset for Research on Accurate Indoor Positioning. Data 2019, 4, 12. [CrossRef]

33. Aranda, F.J.; Parralejo, F.; Álvarez, F.J.; Torres-Sospedra, J. Multi-Slot BLE Raw Database for Accurate Positioning in Mixed Indoor/Outdoor Environments. Data 2020, 5, 67. [CrossRef]

34. Aranda, F.J.; Parralejo, F.; Álvarez, F.J.; Torres-Sospedra, J. Multi-slot BLE Raw Database for Accurate Positioning in Mixed Indoor/Outdoor Environments. Zenodo Repository. 2020. Available online https: / / zenodo.org/record/3927588 (accessed on 23 November 2020).

35. Baronti, P.; Barsocchi, P.; Chessa, S.; Mavilia, F.; Palumbo, F. Indoor Bluetooth Low Energy Dataset for Localization, Tracking, Occupancy, and Social Interaction. Sensors 2018, 18, 4462. [CrossRef] [PubMed]

36. Fukuchi, C.; Fukuchi, R.; Duarte, M. A public dataset of overground and treadmill walking kinematics and kinetics in healthy individuals. PeerJ 2018, 6, e4640. [CrossRef] [PubMed]

37. Schreiber, C.; Moissenet, F. A multimodal dataset of human gait at different walking speeds established on injury-free adult participants. Sci. Data 2019, 6, 111. [CrossRef] [PubMed]

38. Voss, S.; Joyce, J.; Biskis, A.; Parulekar, M.; Armijo, N.; Zampieri, C.; Tracy, R.; Palmer, S.; Fefferman, M.; Ouyang, B.; et al. Normative database of spatiotemporal gait parameters using inertial sensors in typically developing children and young adults. Gait Posture 2020, 80. [CrossRef]

39. Luo, Y.; Coppola, S.; Dixon, P.; Li, S.; Dennerlein, J.; Hu, B. A database of human gait performance on irregular and uneven surfaces collected by wearable sensors. Sci. Data 2020, 7, 219. [CrossRef]

40. Chapron, K.; Bouchard, K.; Gaboury, S. Real-time gait speed evaluation at home in a multi residents context. In Multimedia Tools and Applications; Springer: Berlin/Heidelberg, Germany, 2020.

41. Lohan, E.; Torres-Sospedra, J.; Leppäkoski, H.; Richter, P.; Peng, Z.; Huerta, J. Wi-Fi Crowdsourced Fingerprinting Dataset for Indoor Positioning. Data 2017, 2, 32. [CrossRef]

42. Mendoza-Silva, G.; Richter, P.; Torres-Sospedra, J.; Lohan, E.; Huerta, J. Long-Term WiFi Fingerprinting Dataset for Research on Robust Indoor Positioning. Data 2018, 3, 3. [CrossRef]

43. Tóth, Z. ILONA: Indoor Localization and Navigation System. J. Locat. Based Serv. 2016, 10, $285-302$. [CrossRef]

44. Sikeridis, D.; Papapanagiotou, I.; Devetsikiotis, M. CRAWDAD Dataset Unm/Blebeacon (v.2019-03-12). CRAWDAD Wireless Network Data Archive 2019. Available online: https:/ /crawdad.org/unm/blebeacon/ (accessed on 9 July 2020).

45. zoball. zoball/BLE-Tracking-with-Deep-Learning. 2020. Available online: https://github.com/zoball/BLETracking-with-Deep-Learning (accessed on 9 July 2020).

46. Lohan, E.; Torres-Sospedra, J.; Leppäkoski, H.; Richter, P.; Peng, Z.; Huerta, J. Wi-Fi Crowdsourced Fingerprinting Dataset for Indoor Positioning. Zenodo Repository. 2017. Available online: https://zenodo. org/record/8897981 (accessed on 9 July 2020). [CrossRef]

47. Lohan, E.; Torres-Sospedra, J.; Leppäkoski, H.; Richter, P.; Peng, Z.; Huerta, J. UJIIndoorLoc: A New Multi-Building and Multi-Floor Database for WLAN Fingerprint-Based Indoor Localization Problems. Indoorlocplatform. 2014. Available online: http://indoorlocplatform.uji.es/databases/all/ (accessed on 9 July 2020). 
48. Baronti, P.; Barsocchi, P.; Chessa, S.; Mavilia, F.; Palumbo, F. Indoor Bluetooth Low Energy Datasetfor Localization, Tracking, Occupancy,and Social Interaction. 2018. Available online: http://wnlab.isti.cnr.it/ _media/dataset.zip (accessed on 9 July 2020).

49. Mendoza-Silva, G.M.; Torres-Sospedra, J.; Huerta, J.; Matey Sanz, M. BLE RSS Meaurements Database and Supporting Materials. Zenodo Repository. 2018. Available online: https://zenodo.org/record/1066041 (accessed on 9 July 2020). [CrossRef]

50. Fukuchi, C.; Fukuchi, R.; Duarte, M. A Public Dataset of Overground and Treadmill Walking Kinematics And Kinetics in Healthy Individual. Figshare Repository. 2018. Available online: https://figshare.com/ or https: / / figshare.com/articles / A_public_data_set_of_overground_and_treadmill_walking_kinematics_ and_kinetics_of_healthy_individuals/5722711/2 (accessed on 3 November 2020). [CrossRef]

51. Voss, S.; Joyce, J.; Biskis, A.; Parulekar, M.; Armijo, N.; Zampieri, C.; Tracy, R.; Palmer, S.; Fefferman, M.; Ouyang, B.; et al. Normative Database of Spatiotemporal Gait Parameters Using Inertial Sensors in Typically Developing Children and Young Adults. ScientificDirect, Elsevier, Gait and Posture. Available online: https:/ / www.sciencedirect.com/science/article/pii/S0966636220301600 (accessed on 23 November 2020).

52. Team, S.D.C. Metadata Record for: A Database of Human Gait Performance on Irregular and Uneven Surfaces Collected by Wearable Sensors. 2020. Available online: https:/ / doi.org/10.6084/m9.figshare.12505022.v1 (accessed on 6 December 2020). [CrossRef]

53. LIARALab. Available online: https://github.com/LIARALab (accessed on 3 November 2020).

54. iBKS 105 - Accent Systems. Available online: https://accent-systems.com/product/ibks-105/ (accessed on 3 November 2020).

55. iBKS Plus - Accent Systems. Available online: https://accent-systems.com/product/ibks-plus/ (accessed on 3 November 2020).

56. Furset, K.; Hoffman, P. High Pulse Drain Impact on CR2032 Coin Cell Battery Capacity. Nordic Semiconductor and Energizer; Technical Report, Technical Memo. 2011. Available online: https://www.dmcinfo.com/Portals/ 0/Blog\%20Files /High\%20pulse\%20drain\%20impact\%20on\%20CR2032\%20coin\%20cell\%20battery\% 20capacity.pdf (accessed on 6 December 2020).

57. Goldsmith, A. Wireless Communications; Cambridge University Press: Cambridge, CA, USA, USA, 2005.

Publisher's Note: MDPI stays neutral with regard to jurisdictional claims in published maps and institutional affiliations.

(C) 2020 by the authors. Licensee MDPI, Basel, Switzerland. This article is an open access article distributed under the terms and conditions of the Creative Commons Attribution (CC BY) license (http://creativecommons.org/licenses/by/4.0/). 